\title{
Resolving 45 pm with 300 kV Aberration Corrected STEM
}

\author{
H. Sawada ${ }^{1}$, N. Shimura ${ }^{1}$, K. Satoh ${ }^{1}$, E. Okunishi ${ }^{1}$, F. Hosokawa ${ }^{1}$, N. Shibata ${ }^{2}$, and Y. Ikuhara ${ }^{2}$ \\ ${ }^{1}$ JEOL Ltd., 3-1-2 Musashino, Akishima, Tokyo, 196-8558, Japan. \\ 2.Institute of Engineering Innovation, The University of Tokyo, Tokyo 113-8656 Japan.
}

Eternal challenge towards better resolution has been running by scientists who pursue to see smaller scale world, and countless efforts were spent since the microscope was invented. Within recent decades, Cs corrected microscope [1-3] realized sub-angstrom resolution [4,5]. And further resolution, sub-50-pm was demonstrated in dark-field STEM at $300 \mathrm{kV}$ with an aberration corrected microscope using a Ge [114] specimen [6,7], which shows $47 \mathrm{pm}$ spacing between Ge-Ge atomic columns. Our challenge to get better resolution over the $47 \mathrm{pm}$ was successful with a developed $300 \mathrm{kV}$ aberration corrected microscope equipped with a cold field emission gun (CFEG). This paper reports the results of our challenge, which showed 45 pm resolution using a Si [114] specimen [8].

The microscope we developed is equipped with an asymmetry type spherical aberration corrector [3] in the probe forming lens system. After the Cs correction, the next aberration to block the resolution limit is chromatic aberration. Therefore, the coefficient of chromatic aberration for the probe-forming system is optimized to be $1.35 \mathrm{~mm}$ with a developed objective lens pole piece. With $\Delta \mathrm{E}=0.4 \mathrm{eV}$, simulated probe sizes as a function of Gaussian probe size on a specimen is shown in Fig. 1 (a). Figure 1 (a) indicates that Gaussian probe size, which is demagnified source size, is not negligible to pursue the sub-50 pm resolution. In the observation of $\mathrm{Si}$ [114], a probe current was set to be $3 \mathrm{pA}$. In this condition of probe forming system, the Gaussian probe size on a specimen was estimated to be $7 \mathrm{pm}$. Figure 1 (b) is a profile of the simulated probe with the experimental conditions, showing that Cs corrected STEM at $300 \mathrm{kV}$ with a sufficiently small Gaussian probe size produces a sub-50-pm-sized probe. A full width of half maximum (FWHM) is calculated to be $32.2 \mathrm{pm}$, D50 is $38.6 \mathrm{pm}$ and D59 is $45.9 \mathrm{pm}$. D50 and D59 denote the diameter of the electron beam that includes $50 \%$ and $59 \%$ of the total beam current, respectively.

Before testing the ultimate performance of the microscope by sub-50-pm imaging, we observed $\mathrm{Si}$ [110], Ge [112], Si [112], GaN [211] crystalline specimens, which show distances between atomic column dumbbells of $136 \mathrm{pm}, 82 \mathrm{pm}, 78 \mathrm{pm}$, and $63 \mathrm{pm}$, respectively in dark-field STEM mode. These atomic dumbbells are clearly resolved in raw images as shown in Figs. 2 (a-d). Ge [114] was also imaged to confirm sub-50 pm resolution as shown in Fig. 2 (e). Figure 2(f) shows experimentally obtained HAADF image of Si [114]. Since weaker scattering by $\mathrm{Si}$ atoms than $\mathrm{Ge}$ made a worse single-to-noise ratio in the raw image during the experiment, we doubly increased the dwell time per a pixel in the image acquisition for $\mathrm{Si}$ [114] relative to one for $\mathrm{Ge}[114]$.

Figure 3 (a) shows an experimentally obtained raw dark field image of Si [114], and Figure 3 (b) shows its low pass filtered image. Simulated images for a $20 \mathrm{~nm}$ thick sample were inserted at the lower right in both images. A spot corresponding with $(45 \mathrm{pm})^{-1}$ is confirmed in a Fourier transform shown in Fig. 3 (c). An intensity profile in Fig. 3 (d) also shows 45 pm separation. 


\section{References:}

[1] M Haider et al, Nature 392 (1998), p. 768.

[2] O L Krivanek et al, Ultramicroscopy 78 (1999), p. 1.

[3] F Hosokawa et al, Microscopy 62 (2013), p. 23

[4] P D Nellist et al, Science 305 (2004), p. 1741.

[5] H Sawada et al, Jpn. J.Appl. Phys. 46 (2007), p. L568.

[6] H Sawada et al, J. Electron Microsc. 58, (2009), p. 357.

[7] R. Erni et al, Rev. Lett. 102 (2009), p. 096101.

[8] M. O'Keefe et al, J. Electron Microsc. 54 (2005), p. 169

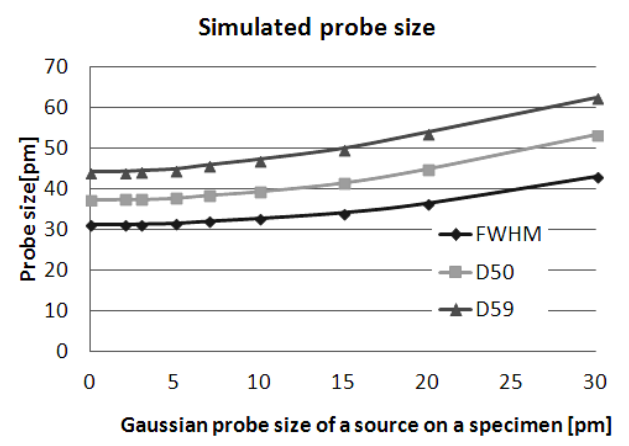

(a)

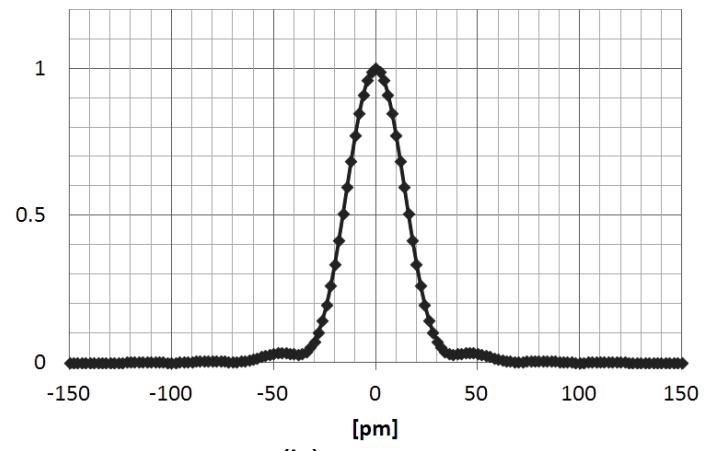

(b)

Figure 1. (a) Simulated probe size including aberrations and Gaussian probe size, depending on Gaussian probe size at $300 \mathrm{kV}$. (b) Profile of the simulated probe with the experimental conditions: $\Delta \mathrm{E}=0.4 \mathrm{eV}, \mathrm{Cc}=1.35 \mathrm{~mm}$.

(a)

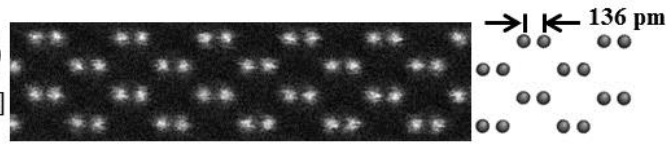

(b)

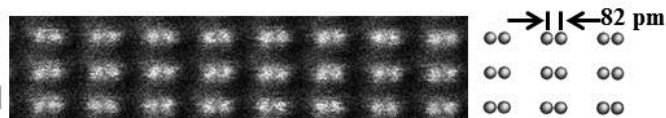

(c)

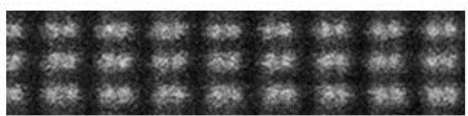

$\rightarrow 0_{00} \leftarrow \leftarrow_{00}^{78 ~ p m}$ Si [112]

(d)

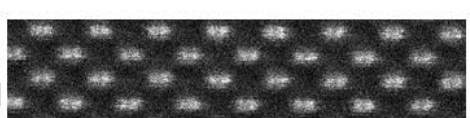

(e)



(f) Si [114]

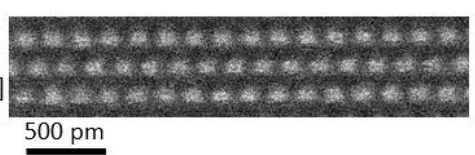

000000

000000

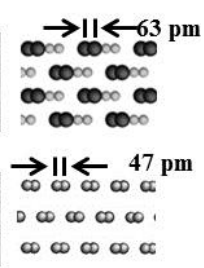

$\vec{\infty} \underset{\infty \infty \mathrm{c}}{\longrightarrow} 45 \mathrm{pm}$ $D \infty \infty \omega \infty$ $\infty \infty \infty \infty a$

Figure 2. (a-f) Raw dark field STEM images and their structural models.
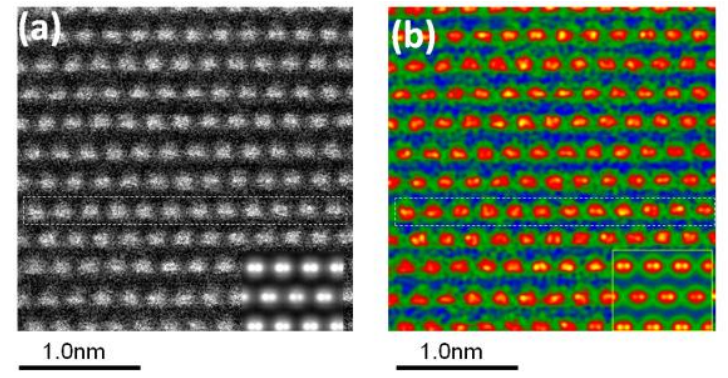

(c)



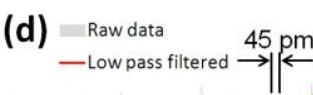



Figure 3. (a) Raw dark field STEM image of Si [114].

(b) Low pass filtered image of (a). Simulated images were inserted at the lower right in both images using the condition in Fig. 1 (b). (c) Power spectrum of (a). (d) Intensity profiles from the dotted rectangles in (a) and (b). 